\title{
Evaluation of Oxidative-Stress Pathway and Recovery of Sudden Sensorineural Hearing Loss
}

\author{
Thaís Gomes Abrahão Elias ${ }^{10}$ Rafael da Costa Monsanto ${ }^{10}$ Jonatas Bussador do Amaral ${ }^{10}$ \\ Lila Missae Oyama2 ${ }^{2}$ Paloma Korehisa Maza ${ }^{2}$ Norma de Oliveira Penido ${ }^{10}$ \\ 1 Department of Otolaryngology, Escola Paulista de Medicina, Universidade \\ Federal de São Paulo (EPM/UNIFESP), São Paulo, SP, Brazil \\ Address for correspondence Thaís Gomes Abrahão Elias, MD, Rua \\ 2 Department of Nutrition Physiology, Escola Paulista de Medicina, \\ Universidade Federal de São Paulo (EPM/UNIFESP), São Paulo, SP, Brasil \\ Pedro de Toledo 947, Vila Clementino, São Paulo, Brazil \\ (e-mail: tabrahao7@gmail.com).
}

Int Arch Otorhinolaryngol 2021;25(3):e428-e432.

\begin{abstract}
Keywords

- sudden hearing loss

- oxidative stress

- prognosis

Introduction Although the pathogenesis of sudden sensorineural hearing loss (SSNHL) has been discussed in the literature, many unclear points remain. Several authors have hypothesized that oxidative stress plays a role in the pathogenesis of noise-related hearing loss, as well as in drug- and aging-related hearing loss. Reactive oxygen species (ROS) may contribute to the pathogenesis of SSNHL in a similar way as in cases of ototoxicity, noise-induced hearing loss and presbyacusis.

Objective The aim of the present study was to find potential peripheral biomarkers to show the levels of oxidative stress in samples of peripheral blood collected from SSNHL patients with and withouth metabolic disease.

Methods In total, 80 consecutive patients with SSNHL were evaluated in the otolaryngology emergency room and outpatient clinic of a tertiary hospital between May 2017 and May 2019. All patients underwent detailed anamnesis, physical examination, audiometry, magnetic resonance imaging (MRI) of the inner ears, and blood tests for serum lipids and plasma activity of thiobarbituric acid reactive species (TBARS).

Results No significant effect of malondialdehyde (MDA) activity was observed regarding the hearing recovery of patients who developed SSNHL.

Conclusion We did not observe a significant correlation between the concentration of TBARs in the peripheral blood or the presence of arterial hypertension and the severity of the initial hearing loss or the prognosis of hearing recovery in patients with SSNHL. The concentration of TBARs in the peripheral blood may not adequately represent the abnormalities that occur in the intracoclear environment.
\end{abstract}

\section{Introduction}

Sudden sensorineural hearing loss (SSNHL) is a challenging clinical issue. Worldwide, the incidence of SSNHL is estimated at 5 to 20 cases per 100 thousand people per year. ${ }^{1}$ The clinical course of SSNHL is the perception of a rapid-onset ( $\leq 72$ hours) hearing loss, which may be unilateral (more frequent) or bilateral. ${ }^{2}$ In the majority of the cases, the etiology is not identified, in spite of comprehensive clinical, laboratory, and imaging evaluations, and the condition is then termed idiopathic SSNHL. ${ }^{2}$ The pathogenesis, the optimal treatments, and the prognostic received

February 10, 2020

accepted

June 5, 2020

published online

September 30, 2020
DOI https://doi.org/ 10.1055/s-0040-1714130. ISSN 1809-9777.

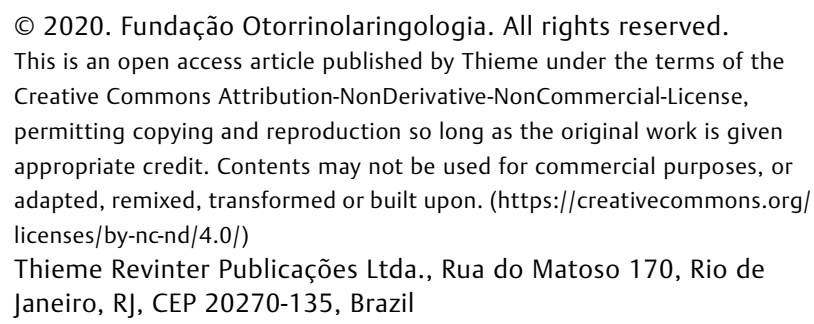


factors of SSNHL are still under debate in the scientific community. 3,4

Several authors hypothesized that oxidative stress plays a role in the pathogenesis of noise-induced hearing loss, as well as in drug- and aging-related hearing loss. ${ }^{5-7}$ Oxidative stress occurs due to a dysregulation in the cellular redox activity in which the production of reactive oxygen species (ROS) outweighs the clearance of those ROS by endogenous antioxidant enzymes and molecular chaperones. ${ }^{8}$ The generation of ROS slowly impairs the functioning of mitochondria, ultimately resulting in damage to biological molecules (such as DNA). ${ }^{9}$ Elevated levels of ROS cause subsequent apoptosis of cells through several pathways, including oxidative phosphorylation dysfunction, increased pro-ROS enzyme activity, and decreased anti-ROS activity. ${ }^{10,11}$

Previous studies suggested that redox imbalance might be associated with the pathogenesis of hypertension. ${ }^{12,13}$ Reactive oxygen species are known to modulate numerous pathways that are important for the control of systemic vascular resistance and blood pressure, including decreased biovailability of nitric oxide, inflammation, imbalance in salt and water homeostasis, hyperactivity of the sympathetic nervous system, and disturbances of the renin-angiotensinaldosterone-system. ${ }^{14,15}$

One study ${ }^{13}$ has shown a correlation of oxidative stress with metabolic diseases, among which are excess weight (obesity/overweight), diabetes, hypertension, and dyslipidaemia. Reactive oxygen species impact insulin signaling pathways, thus leading to an insulin-resistant state, increased hepatic influx of free fatty acids, increased lipogenesis, as well as increased triglyceride storage, inducing hepatocyte dysfunction or death. ${ }^{16}$

Therefore, we hypothesize that ROS may contribute to the pathogenesis of SSNHL in a similar way as in cases of ototoxicity, noise-induced hearing loss, and presbyacusis. A better understanding of the complex role of oxidative stress in the pathogenesis of SSNHL might reveal new targets for prognostic factors and therapeutic modification. The aim of the present study was to find potential peripheral biomarkers to show the levels of oxidative stress in samples of peripheral blood collected from SSNHL patients with and withouth metabolic disease.

\section{Methods}

In total, 80 consecutive patients who developed SSNHL were evaluated in the otolaryngology emergency room and outpatient clinic of a tertiary hospital between May 2017 and May 2019, regardless of age group. Sudden sensorineural hearing loss was defined as the presence of a rapid-onset ( $\leq 72$ hours) hearing loss of at least $30 \mathrm{~dB}$ in 3 contiguous frequencies. Only previously-untreated cases were included in our casuistic. Written informed consent was obtained from all participants. The present study was approved by the Ethics in Research Committee of Escola Paulista de Medicina/Universidade Federal de São Paulo (EPM/UNIFESP) under protocol number 2.149.364.

All patients underwent detailed anamnesis, physical examination, audiometry, magnetic resonance imaging (MRI) of the inner ears, and blood tests for serum lipids and plasma activity of thiobarbituric acid reactive species (TBARS).

The inclusion criteria for the diagnosis of diabetic patients were: having received a confirmed diagnosis of type- 1 or type-2 diabetes mellitus; and being under treatment with insulin or glucose-lowering oral medications.

The inclusion criteria for the diagnosis of hypertensive patients were: having systolic blood pressure $\geq 140 \mathrm{mmHg}$ and/or diastolic blood pressure $\geq 90 \mathrm{mmHg}$, measured by a physician according to reported methods on at least 3 visits; and having been submitted to a previous treatment for hypertension.

We excluded patients who were evaluated after 14 days since the beginning of the hearing loss, those who had less than $25 \mathrm{~dB}$ in the initial hearing test, and those who had recurrent hearing loss. All patients were treated with prednisolone $1 \mathrm{mg} / \mathrm{Kg} /$ day (maximum dose of $60 \mathrm{mg} /$ day) once daily for at least 14 days. The patients underwent weekly audiograms (performed by the same professional and using the same equipment), and, in cases in which the audiometry showed improvements as compared with the one performed one week earlier (a difference of at least $10 \mathrm{~dB}$ between two sequential audiometries). A significant improvement in the audiometry was defined as an improvement of $10 \mathrm{dBHL}$ or more in pure-tone average (PTA), or an improvement of $10 \%$ or more in the speech discrimination score (SDS). Then, the dose of prednisolone was tapered for 3 weeks until complete withdrawal. Equivalent doses of deflazacort (maximum dose of $90 \mathrm{mg}$ /day) were administered to patients who did not tolerate prednisone or who had arterial hypertension or diabetes.

The degree of initial hearing loss was classified according to the PTA at $250 \mathrm{~Hz}, 500 \mathrm{~Hz}, 1,000 \mathrm{~Hz}, 2,000 \mathrm{~Hz}, 4,000 \mathrm{~Hz}$ and $8,000 \mathrm{~Hz}$ as: 1 ) mild ( $26 \mathrm{~dB}$ to $40 \mathrm{~dB}) ; 2$ ) moderate ( $41 \mathrm{~dB}$ to $55 \mathrm{~dB}$ ); 3) moderate-severe ( $56 \mathrm{~dB}$ to $70 \mathrm{~dB}$ ); 4) severe $(71 \mathrm{~dB}$ to $90 \mathrm{~dB})$; and 5$)$ profound $(91 \mathrm{~dB}) .{ }^{17}$ The success of the treatment was assessed based on the hearing gains in the PTA and SDS as per the 2019 clinical practice guideline on sudden hearing loss (American Academy of OtolaryngologyHead and Neck Surgery): ${ }^{17}$ an improvement $<10 \mathrm{~dB}$ HL in the PTA was defined as no recovery (group 1); an improvement $\geq 10 \mathrm{~dB}$ HL in the PTA or an improvement $\geq 10 \%$ in the SDS was defined as partial recovery (group 2); and an improvement within the $10 \mathrm{~dB}$ HL range of the unaffected ear's hearing threshold and within $5 \%$ to $10 \%$ of its SDS was defined as complete recovery (group 3 ).

The concentration of malondialdehyde (MDA), an index of lipid peroxidation, was determined based on the production of thiobarbituric acid reactive species (TBARS). The concentrations of MDA were calculated using a TBARS assay kit (R\&D Systems, Inc., Minneapolis, MN, US) in accordance with the manufacturer's instructions, which was normalized to protein concentration. In brief, equal volumes $(100 \mu \mathrm{L})$ of samples and SDS were added to a $5-\mathrm{mL}$ conical vial. After vortex mixing, the samples were mixed with $0.4 \mathrm{~mL}$ of $1 \%$ thiobarbituric acid in $50 \mathrm{~mm} \mathrm{NaOH}$ and $0.2 \mathrm{~mL}$ (20\%) $\mathrm{H}_{3} \mathrm{PO}_{4}$. The mixture was heated to $100^{\circ} \mathrm{C}$ for 15 minutes. After 10 minutes of incubation on ice, the vials 
Table 1 Responses to the treatment for sudden sensorineural hearing loss in patients with and without high levels of total cholesterol

\begin{tabular}{|c|c|c|c|c|c|c|c|}
\hline & & \multicolumn{4}{|c|}{ High levels of total cholesterol } & \multirow{2}{*}{\multicolumn{2}{|c|}{ Total }} \\
\hline & & \multicolumn{2}{|c|}{ Withouth } & \multicolumn{2}{|c|}{ With } & & \\
\hline & & $\mathrm{N}$ & $\%$ & $\mathrm{~N}$ & $\%$ & $\mathrm{~N}$ & $\%$ \\
\hline \multirow[t]{3}{*}{ Recovery of hearing loss } & Total recovery & 21 & $31.3 \%$ & 2 & $15.4 \%$ & 23 & $28.8 \%$ \\
\hline & Partial recovery & 26 & $38.8 \%$ & 3 & $23.1 \%$ & 29 & $36.3 \%$ \\
\hline & No improvement & 20 & $29.9 \%$ & 8 & $61.5 \%$ & 28 & $35.0 \%$ \\
\hline \multicolumn{2}{|l|}{ Total } & 67 & $100.0 \%$ & 13 & $100.0 \%$ & 80 & $100.0 \%$ \\
\hline
\end{tabular}

were centrifuged at $1,600 \times \mathrm{g}$ for 10 minutes at $4^{\circ} \mathrm{C}$. The samples $(100 \mu \mathrm{L})$ were loaded into 96 -well assay plates, and the absorbance of each well was measured at $540 \mathrm{~nm}$ using a microplate reader.

The level of total cholesterol was analyzed using a commercial kit (Labtest, Lagoa Santa, Minas Gerais, Brazil). The protein levels were measured using the Bradford method.

For the statistical analyses, the three groups were compared using the Chi-squared test, the Kruskal-Wallis test, analysis of variance (ANOVA), and the Spearman correlation test using the Statistical Package for the Social Sciences (SPSS, IBM Corp., Armonk, NY, US) software, version 20.0. Values of $p<0.05$ were considered statistically significant.

\section{Results}

\section{Demography}

A total of 80 patients were included in the presente study. The mean age of the study population was 48.6 years (range: 7-89 years). There was a slight predominance of female subjects: 1.8:1 (female:male). At the time of the first evaluation, 23 patients $(28.75 \%)$ had systemic arterial hypertension, 13 patients (16.25\%) had high levels of total cholesterol, and 10 patients (12.5\%) had diabetes.

\section{Audiologic Test}

The degree of initial sensorineural hearing loss was: 2 patients (2.5\%) with mild hearing loss; 29 patients (36.25\%) with moderate hearing loss; 35 patients (43.75\%) with severe hearing loss; and 14 patients (17.5\%) with profound hearing loss.

Thirty days after the treatment, we observed that 23 patients (28.75\%) achieved total recovery of the hearing loss (group 1), 29 patients (36.25\%) had partial recovery of the hearing loss (group 2), and 28 patients (35\%) did not recover from the initial hearing loss (group 3). We observed a significant positive correlation between the degree of initial sensorineural hearing loss and the recovery of the hearing loss.

\section{Association between Age and Recovery of Hearing Loss}

We observed a significant positive correlation between the age of the patients and the success of the treatment (KruskalWallis test; $p=0.014$ ).

\section{Association between Comorbidities and Recovery of Hearing Loss}

We did not observe a significant association between the presence of systemic arterial hypertension and the recovery of the hearing (Cui-squared test; $p=0,926$ ). In contrast, we observed that the degree of recovery was significantly worse in patients with high levels of total cholesterol and diabetes as compared with patients who did not have those comorbidities ( $\mathbf{- T a b l e s} \mathbf{1}$ and $\mathbf{2}$ ).

\section{Association between the Indexes of Oxidative Stress and Recovery of Hearing Loss}

The activity of MDA was measured in the plasma of the patients. These data were also reflected in a quantitative TBARS assay. No significant effect of MDA activity was observed among the three study groups (-Table $\mathbf{3}$ ).

\section{Discussion}

To date, the etiology of SSHNL remains unknown, as well as which treatment is optimal and the prognostic markers. Although some authors ${ }^{18}$ describe that $25 \%$ of the patients achieve complete recovery of the hearing following SSNHL, it

Table 2 Responses to the treatment for sudden sensorineural hearing loss in patients with and without diabetes

\begin{tabular}{|c|c|c|c|c|c|c|c|}
\hline & & \multicolumn{4}{|c|}{ Diabetes } & \multirow{2}{*}{\multicolumn{2}{|c|}{ Total }} \\
\hline & & \multicolumn{2}{|c|}{ Withouth } & \multicolumn{2}{|c|}{ With } & & \\
\hline & & $\mathrm{N}$ & $\%$ & $\mathbf{N}$ & $\%$ & $\mathrm{~N}$ & $\%$ \\
\hline \multirow[t]{3}{*}{ Recovery of hearing loss } & Total recovery & 22 & $31.4 \%$ & 1 & $10.0 \%$ & 23 & $28.8 \%$ \\
\hline & Partial recovery & 27 & $38.6 \%$ & 2 & $20.0 \%$ & 29 & $36.3 \%$ \\
\hline & No improvement & 21 & $30.0 \%$ & 7 & $70.0 \%$ & 28 & $35.0 \%$ \\
\hline \multicolumn{2}{|l|}{ Total } & 70 & $100.0 \%$ & 10 & $100.0 \%$ & 80 & $100.0 \%$ \\
\hline
\end{tabular}


Table 3 Association between the activity of thiobarbituric acid reactive species and the responses to the treatment for sudden sensorineural hearing loss

\begin{tabular}{|c|c|c|c|c|}
\hline \multirow[t]{2}{*}{ Thiobarbituric acid reactive species $(\mathrm{nmol} / \mathrm{mL})$} & \multicolumn{3}{|c|}{ Recovery of hearing loss } & \multirow[b]{2}{*}{ Kruskal-Wallis $(p)$} \\
\hline & Total & Partial & Without recovery & \\
\hline Average & 0.831 & 0.788 & 0.776 & \\
\hline Median & 0.688 & 0.763 & 0.735 & 0.735 \\
\hline Standard deviation & 0.329 & 0.170 & 0.248 & \\
\hline $\mathrm{n}$ & 23 & 29 & 28 & \\
\hline
\end{tabular}

has been demonstrated that $50 \%$ of the patients with SSNL only achieve partial recovery, and, in the remaining $25 \%$, the hearing will not improve after the initial insult at all. ${ }^{18} \mathrm{~A}$ review of the temporal bones from the collection of the Massachusetts Eye and Ear Infirmary ${ }^{19}$ showed that the most common pathological findings in temporal bones from donors who had SSNHL were loss of hair and supporting cells in the organ of Corti. Histopathologically, most innerear diseases that affect the cochlea result in very similar patterns of degeneration; therefore, it was not possible to infer the potential pathophysiological mechanisms that could have resulted in those findings. ${ }^{19}$

Oxidative stress induced by ROS and disruption of the redox status have been reported to play a pivotal role in the cardiovascular, cerebrovascular and inner-ear systems. ${ }^{20}$ Several inner-ear pathologies, including acoustic trauma, SSNHL, presbyacusis and Meniere disease are hypothesized to involve dysfunction of the microvascular circulation. ${ }^{21}$ In the cochlea, the role of ROS in the onset and progression of damage has been further supported by the observation of increased expression of ROS in the cochlea following exposure to damaging levels of noise, well before morphological signs of damage are detected, and they persist for 7 to 10 days after exposure. 8,22

It is established that, in patients with noise-induced hearing loss, there is an overexpression of ROS in the cochlea, resulting in oxidative stress. ${ }^{23}$ Excessive generation of free radicals induced by noise exposure causes the accumulation of lipid peroxidation products, oxidized proteins, and oxidative DNA damage in the cochlea, resulting in death of the hair cells and nerve endings, and subsequent hearing loss. ${ }^{6,24}$ In the present study, no statistically significant changes in the concentration of TBARS in the plasma of the patients in all three groups were observed. To our knowledge, no previous study has evaluated the concentration of TBARs and MDA in the peripheral blood in patients with SSNHL. Notwithstanding our negative findings, several studies ${ }^{6-11}$ have shown that endothelial dysfunction secondary to the accumulation of ROS might play an important role in the pathophysiology of SSNHL. Capaccio et $\mathrm{al}^{25}$ demonstrated that indicators of oxidative stress increased after the development of SSNHL, and they suggested that - in addition to the traditional risk factors for ischemia - oxidative stress is a risk factor for endothelial injury in the terminal microvascular circulation. ${ }^{26}$ The presence of ROS within the cochlear tissue raises the issue of whether the ROS lead to cochlear damage or if the increase in the expression of ROS occur secondary to an acute injury to the cochlear cells. However, our findings do not support the hypothesis that measuring the concentration of TBARs and MDA in the peripheral blood could be used as a prognosis indicator in patients with SSNHL. Our data have some limitation. First, the samples of blood were taken from the systemic circulation to measure the oxidative stress. Additionally, we used just one product of ROS. It is still unclear what effect ROS might have on SSNHL.

We observed that the presence of diabetes and dyslipidemia associated with worse hearing prognosis in patients with SSNHL as compared with people without those comorbidities, since the patients with diabetes and dyslipidemia had worse final PTAs and higher rates of partial or no improvement in the hearing levels. Those results may be explained by several previous observations from the literature. The cochlea is an end organ, which is metabolically dependent of nutrient and oxygen supply to maintain its normal physiological function. Thus, it is very sensitive to alterations in blood circulation. ${ }^{27}$ Lipid metabolic disorders may contribute to cochlear ischemia due to increased blood viscosity and increased thickness of the endothelium of the terminal blood vessels through several mechanisms, including the formation of atheromatous plaques, endothelial dysfunction, and vascular inflamation. ${ }^{8,28-30}$ In this regard, a study ${ }^{31}$ including 94 patients with SSNHL showed a significant negative correlation between hearing recovery and total cholesterol levels. Diabetes may also induce cochlear microangiopathy, resulting in SSNHL and reducing the rate of recovery from this condition. Because atrophy of the spiral ganglion and demyelination of the eighth cranial nerve have been observed in individuals with diabetes, this type of diabetes-induced neuropathy may cause SSNHL. ${ }^{32}$

We did not observe a significant correlation between arterial hypertension and hearing prognosis following SSNHL. Althouth several previous studies ${ }^{33-35}$ demonstrated that hypertension was considered a poor prognostic factor, for it could be associated with microvascular dysfunction in the cochlea, our findings do not support those assumptions.

While there have been some advances in the hypothesis of a common final pathway of insult for hearing loss in patients with SSNHL, there is insufficient evidence supporting the use of biological markers as indicators of hearing prognosis in SSNHL. ${ }^{36}$ In the present study, we planned to use TBARS as a biomarker to detect patients with worse prognoses earlier during the onset of hearing loss. However, we did not observe 
a correlation between the concentration of TBARS and the severity of the initial hearing loss or the prognosis of hearing recovery in patients with SSNHL. We hypothesize that considering that the inner ear is located in the hardest bone in the body, making its direct acess difficult - the concentration of TBARs in the peripheral blood may not adequately represent the abnormalities that occur in the intracoclear environment. ${ }^{36}$

\section{Conclusion}

We did not observe a significant correlation between the concentration of TBARs in the peripheral blood or the presence of arterial hypertension in the severity of the initial hearing loss or the prognosis of hearing recovery in patients with SSNHL. But we observed that the presence of dyslipidemia or diabetes is associated with poorer hearing prognosis in patients with SSNHL. Patients with diabetes and dyslipidemia had worse final PTAs and higher rates of partial or no improvement in the hearing levels as compared with people without those comorbidities.

\section{Conflict of Interests}

The authors have no conflicts of interest to declare.

\section{References}

1 Rauch SD. Clinical practice. Idiopathic sudden sensorineural hearing loss. N Engl J Med 2008;359(08):833-840

2 Schreiber BE, Agrup C, Haskard DO, Luxon LM. Sudden sensorineural hearing loss. Lancet 2010;375(9721):1203-1211

3 Cole RR, Jahrsdoerfer RA. Sudden hearing loss: an update. Am J Otol 1988;9(03):211-215

4 Wen YH, Chen PR, Wu HP. Prognostic factors of profound idiopathic sudden sensorineural hearing loss. Eur Arch Otorhinolaryngol 2014;271(06):1423-1429

5 Yamasoba T, Lin FR, Someya S, Kashio A, Sakamoto T, Kondo K. Current concepts in age-related hearing loss: epidemiology and mechanistic pathways. Hear Res 2013;303:30-38

6 Henderson D, Bielefeld EC, Harris KC, Hu BH. The role of oxidative stress in noise-induced hearing loss. Ear Hear 2006;27(01):1-19

7 Evans P, Halliwell B. Free radicals and hearing. Cause, consequence, and criteria. Ann N Y Acad Sci 1999;884:19-40

8 Ohlemiller KK, Wright JS, Dugan LL. Early elevation of cochlear reactive oxygen species following noise exposure. Audiol Neurotol 1999;4(05):229-236

9 Kamogashira T, Fujimoto C, Yamasoba T. Reactive oxygen species, apoptosis, and mitochondrial dysfunction in hearing loss. BioMed Res Int 2015;2015:617207

10 Souza MEDCA, Costa KVTD, Vitorino PA, Bueno NB, Menezes PL. Effect of antioxidant supplementation on the auditory threshold in sensorineural hearing loss: a meta-analysis. Rev Bras Otorrinolaringol (Engl Ed) 2018;84(03):368-380

11 Menardo J, Tang Y, Ladrech S, et al. Oxidative stress, inflammation, and autophagic stress as the key mechanisms of premature agerelated hearing loss in SAMP8 mouse Cochlea. Antioxid Redox Signal 2012;16(03):263-274

12 Montezano AC, Touyz RM. Oxidative stress, Noxs, and hypertension: experimental evidence and clinical controversies. Ann Med 2012;44(Suppl 1):S2-S16

13 Montezano AC, Dulak-Lis M, Tsiropoulou S, Harvey A, Briones AM, Touyz RM. Oxidative stress and human hypertension: vascular mechanisms, biomarkers, and novel therapies. Can J Cardiol 2015; 31(05):631-641
14 Virdis A, Bacca A, Colucci R, et al. Endothelial dysfunction in small arteries of essential hypertensive patients: role of cyclooxygenase-2 in oxidative stress generation. Hypertension 2013;62(02):337-344

15 Vanhoutte PM, Shimokawa H, Tang EH, Feletou M. Endothelial dysfunction and vascular disease. Acta Physiol (Oxf) 2009;196 (02):193-222

16 Savini I, Catani MV, Evangelista D, Gasperi V, Avigliano L. Obesityassociated oxidative stress: strategies finalized to improve redox state. Int J Mol Sci 2013;14(05):10497-10538

17 Chandrasekhar SS, Tsai do BS, Schwartz SR, et al. Clinical practice guideline: sudden hearing loss (update). Otolaryngol Head Neck Surg 2019;161:1-45

18 Jung SY, Shim HS, Hah YM, Kim SH, Yeo SG. Association of metabolic syndrome with sudden sensorineural hearing loss. JAMA Otolaryngol Head Neck Surg 2018;144(04):308-314

19 Merchant SN, Adams JC, Nadol JB Jr. Pathology and pathophysiology of idiopathic sudden sensorineural hearing loss. Otol Neurotol 2005;26(02):151-160

20 Juhn SK, Hunter BA, Odland RM. Blood-labyrinth barrier and fluid dynamics of the inner ear. Int Tinnitus J 2001;7(02):72-83

21 Shi X, Han W, Yamamoto H, et al. The cochlear pericytes. Microcirculation 2008;15(06):515-529

22 Yamane H, Nakai Y, Takayama M, Iguchi H, Nakagawa T, Kojima A. Appearance of free radicals in the guinea pig inner ear after noiseinduced acoustic trauma. Eur Arch Otorhinolaryngol 1995;252 (08):504-508

23 Fujimoto C, Yamasoba T. Oxidative stresses and mitochondrial dysfunction in age-related hearing loss. Oxid Med Cell Longev 2014;2014:582849

24 Wong ACY, Ryan AF. Mechanisms of sensorineural cell damage, death and survival in the cochlea. Front Aging Neurosci 2015;7:58

25 Capaccio P, Pignataro L, Gaini LM, et al. Unbalanced oxidative status in idiopathic sudden sensorineural hearing loss. Eur Arch Otorhinolaryngol 2012;269(02):449-453

26 Böttger EC, Schacht J. The mitochondrion: a perpetrator of acquired hearing loss. Hear Res 2013;303:12-19

27 Chung JW, Shin JE, Han KW, et al. Up-regulation of hypoxia-inducible factor- 1 alpha by cobalt chloride prevents hearing loss in noiseexposed mice. Environ Toxicol Pharmacol 2011;31(01):153-159

28 Ohinata Y, Miller JM, Altschuler RA, Schacht J. Intense noise induces formation of vasoactive lipid peroxidation products in the cochlea. Brain Res 2000;878(1-2):163-173

29 Onal M, Elsurer C, Selimoglu N, et al. Ozone prevents cochlear damage from ischemia-reperfusion injury in Guinea pigs. Artif Organs 2017;41(08):744-752

30 Kim JS, Lopez I, DiPatre PL, Liu F, Ishiyama A, Baloh RW. Internal auditory artery infarction: clinicopathologic correlation. Neurology 1999;52(01):40-44

31 Lee JS, Kim DH, Lee HJ, et al. Lipid profiles and obesity as potential risk factors of sudden sensorineural hearing loss. PLoS One 2015; 10(04): 0122496

32 Quaranta N, Squeo V, Sangineto M, Graziano G, Sabbà C. High total cholesterol in peripheral blood correlates with poorer hearing recovery in idiopathic sudden sensorineural hearing loss. PLoS One 2015;10(07):e0133300

33 Anjos MF, Takata TT, Chaim RM, et al. Idiopathic sudden sensorineural hearing loss: a evolution in the presence of hypertension, diabetes mellitus and dyslipidemia. Rev Bras Otorrinolaringol (Engl Ed) 2010;76(03):363-369

34 Byl FM Jr. Sudden hearing loss: eight years' experience and suggested prognostic table. Laryngoscope 1984;94(5 Pt 1):647-661

35 Harada H, KatoT. Prognosis for sudden sensorineural hearing loss: a retrospective study using logistical regression analysis. Int Tinnitus J 2005;11(02):115-118

36 Ozdamar K, Sen A, Gonel A. Assessment of oxidative stress in patients with sudden hearing loss: a non-randomized prospective clinical study. Indian J Otolaryngol Head Neck Surg 2019;71 (Suppl 2):1543-1548 